\title{
Experimental demonstration of quantum source coding
}

\author{
Yasuyoshi Mitsumori ${ }^{1,2}$, John A. Vaccaro ${ }^{3}$, Stephen M. Barnett ${ }^{4}$, Erika \\ Andersson $^{4}$, Atsushi Hasegawa ${ }^{1,2}$, Masahiro Takeoka ${ }^{1,2}$, and Masahide Sasaki ${ }^{1,2}$ \\ 1 Communications Research Laboratory,Koganei, 4-2-1 Nukuikita, Koganei, Tokyo 184-8795, Japan \\ 2 CREST, Japan Science and Technology Corporation, 3-13-3 Shibuya, Tokyo 150-0002, Japan \\ 3 Department of Physical Science, University of Hertfordshire, College Lane Hatfield AL109AB, U.K. \\ 4 Department of Physics and Applied Physics, University of Strathclyde, Glasgow G4 0NG, Scotland
}

(Dated: February 1, 2008)

\begin{abstract}
We report an experimental demonstration of Schumacher's quantum noiseless coding theorem. Our experiment employs a sequence of single photons each of which represents three qubits. We initially prepare each photon in one of a set of 8 non-orthogonal codeword states corresponding to the value of a block of three binary letters. We use quantum coding to compress this quantum data into a two-qubit quantum channel and then uncompress the two-qubit channel to restore the original data with a fidelity approaching the theoretical limit.
\end{abstract}

PACS numbers: 03.67.Hk, 03.65.Ta, 42.50.-p

Keywords:

\section{Introduction}

The coding of messages is a fundamental issue in information theory. There are two basic coding problems, namely how to represent messages as efficiently as possible and how to transmit messages as precisely as possible. The former is called source coding, and is related practically to data compression, while the latter is called channel coding and is concerned with error correction. All information processing techniques are connected with these two kinds of coding problem. We focus on source coding in this report.

Essentially, source coding entails the coding of common alphabet in a message as short sequences of code letters, such as the binary digits $\{0,1\}$, and uncommon alphabet as longer sequences, to make the average length of the coded message as short as possible. The unequal frequencies of the letters imply a redundancy that enables the compression of the message. Shannon's source coding theorem gives the bounds on the degree a classical message can be compressed. For a source of alphabet $\{A, B, \ldots, Z\}$ with given prior probabilities $\{P(A), P(B), \ldots, P(Z)\}$, the minimum average length of the coded message is given by the Shannon entropy

$$
H=-\sum_{n=A, B, \ldots} P(n) \log _{2} P(n)
$$

$H$ takes its maximum value when all alphabet appear with equal probability, that is, when we know nothing better than a random guess for each element. Then any compression is impossible.

In quantum domain, there is another kind of redundancy when the letters are conveyed by the nonorthogonal quantum states, $\left|\psi_{A}\right\rangle,\left|\psi_{B}\right\rangle,\left|\psi_{C}\right\rangle, \cdots$ with

*Electronic address: J.A.Vaccaro@herts.ac.uk Electronic address: steve@phys.strath.ac.uk Electronic address: psasaki@crl.go.jp corresponding probabilities $P_{A}, P_{B}, P_{C}, \cdots$ Significantly, compression is possible here even if $P_{A}=P_{B}=$ $P_{C}=\cdots$, in contrast to the classical case. Recently Schumacher and Jozsa derived the quantum version of the source coding theorem. The quantum noiseless coding theorem [1, 2] implies that by coding the quantum message in blocks of $K$ letters, $K S(\hat{\rho})$ qubits are necessary to encode each block in the limit $K \rightarrow \infty$, where $S(\hat{\rho})$ is the von Neumann entropy of the density operator $\hat{\rho}=\sum P_{n}\left|\psi_{n}\right\rangle\left\langle\psi_{n}\right|$ representing the average state of the letter states.

In addition to its central role in quantum information theory, the compression of non-orthogonal data sets has significant practical advantages. For example, in longhaul optical communication channels one must deal with sequences of attenuated weak coherent pulses, that is, non-orthogonal states. Expensive quantum channel resources can be saved by compressing the sequences before storing or relaying to another channel.

Given its fundamental as well as practical importance, it is perhaps surprising that quantum source coding has not been demonstrated experimentally to date. We report an experimental demonstration of the reliable communication of 3-qubit codewords over a 2-qubit quantum channel. The minimum resources needed for an analogous classical channel would be 3 bits per codeword.

\section{Quantum coding protocols}

Our demonstration is based on the example given by Jozsa and Schumacher [2]. Imagine Alice needs to send Bob a message composed of an alphabet of 2 letters, "+" and "-", represented by the letter states $\left|\psi_{+}\right\rangle$and $\left|\psi_{-}\right\rangle$,

$$
\left|\psi_{ \pm}\right\rangle=\alpha|0\rangle+\beta_{ \pm}|1\rangle
$$

Here $|0\rangle,|1\rangle$ are an orthonormal (computational) basis, $\beta_{ \pm}= \pm \beta, \alpha^{2}+\beta^{2}=1$, and for clarity we assume $\alpha$ and $\beta$ are real numbers. Let the letter states occur with equal likelihood so that the density operator representing the average letter state is $\hat{\rho}=\alpha^{2}|0\rangle\left\langle 0\left|+\beta^{2}\right| 1\right\rangle\langle 1|$. 
The corresponding von Neumann entropy is $S(\hat{\rho})=$ $-\alpha^{2} \log _{2} \alpha^{2}-\beta^{2} \log _{2} \beta^{2}$.

If the letter states are orthogonal, $\alpha^{2}=\beta^{2}=\frac{1}{2}$, then 1 qubit (or classically 1 bit) is needed to encode each letter faithfully. In this case a sequence of letter states cannot be compressed to a smaller code. However, the von Neumann entropy of $\hat{\rho}$ is 0.4690 bits for the case $\alpha^{2}=0.9$ [2]. According to the quantum noiseless coding theorem, in the limit of large block sizes Alice needs approximately $1 / 2$ qubit per letter state to faithfully transmit the message to Bob.

Following [2] we use blocks of 3 letter states:

$$
\begin{aligned}
\left|B_{\mathbf{L}}\right\rangle= & \left|\psi_{L_{1}}\right\rangle \otimes\left|\psi_{L_{2}}\right\rangle \otimes\left|\psi_{L_{3}}\right\rangle \\
= & \alpha^{3}|000\rangle+\alpha^{2}\left(\beta_{L_{1}}|100\rangle+\beta_{L_{2}}|010\rangle+\beta_{L_{3}}|001\rangle\right) \\
& +\alpha\left(\beta_{L_{1}} \beta_{L_{2}}|110\rangle+\beta_{L_{2}} \beta_{L_{3}}|011\rangle+\beta_{L_{1}} \beta_{L_{3}}|101\rangle\right) \\
& +\beta_{L_{1}} \beta_{L_{2}} \beta_{L_{3}}|111\rangle
\end{aligned}
$$

where $\mathbf{L}=\left(L_{1}, L_{2}, L_{3}\right)$ and $L_{1}, L_{2}$ and $L_{3} \in\{+,-\}$. The index $\mathbf{L}$ selects one of 8 possible letter state configurations. In our quantum coding scheme [3], Alice first applies the unitary transformation $\hat{U}$ which leaves all computational bases states unchanged except for the following mapping $\hat{U}|100\rangle=|011\rangle$ and $\hat{U}|011\rangle=|100\rangle$. The state of a block after the application of $\hat{U}$ is

$\hat{U}\left|B_{\mathbf{L}}\right\rangle=\alpha^{2} \sqrt{1+2 \beta^{2}}|0\rangle \otimes\left|\mu_{\mathbf{L}}\right\rangle+\beta^{2} \sqrt{1+2 \alpha^{2}}|1\rangle \otimes\left|\nu_{\mathbf{L}}\right\rangle$

where

$$
\left|\mu_{\mathbf{L}}\right\rangle=\frac{1}{\sqrt{1+2 \beta^{2}}}\left(\alpha|00\rangle+\beta_{L_{1}}|11\rangle+\beta_{L_{2}}|10\rangle+\beta_{L_{3}}|01\rangle\right)
$$

$$
\begin{aligned}
\left|\nu_{\mathbf{L}}\right\rangle= & \frac{1}{\beta^{2} \sqrt{1+2 \alpha^{2}}}\left[\alpha \left(\beta_{L_{1}} \beta_{L_{2}}|10\rangle+\beta_{L_{1}} \beta_{L_{3}}|01\rangle\right.\right. \\
& \left.\left.+\beta_{L_{2}} \beta_{L_{3}}|00\rangle\right)+\beta_{L_{1}} \beta_{L_{2}} \beta_{L_{3}}|11\rangle\right] .
\end{aligned}
$$

Alice then makes a projection measurement of the first (leftmost) qubit in the computational basis. The last two qubits represent the coded block state sent to Bob. We consider two different protocols corresponding to two different actions Alice takes when the projective measurement results in the state $|1\rangle$. Essentially, the coding protocols amount to a perfect transmission of the most likely parts of letter state configurations, and a less faithful transmission of, or even discarding of, the remaining less likely part.

The first protocol, which we shall label $\mathrm{P}_{1}$, is to treat the projection measurement result $|1\rangle$ as a failure. Under this protocol the state of the 2-qubit quantum channel is

$$
\hat{\rho}_{\mathbf{L}}^{(1)}=\left|\mu_{\mathbf{L}}\right\rangle\left\langle\mu_{\mathbf{L}}\right|
$$

with probability $p=\alpha^{4}\left(1+2 \beta^{2}\right)$ and a state of zero overlap with any block state with probability $1-p$. Bob decodes the state $\hat{\rho}_{\mathbf{L}}^{(1)}$ at his end of the quantum channel by preparing an extra qubit in the state $|0\rangle$ and applying

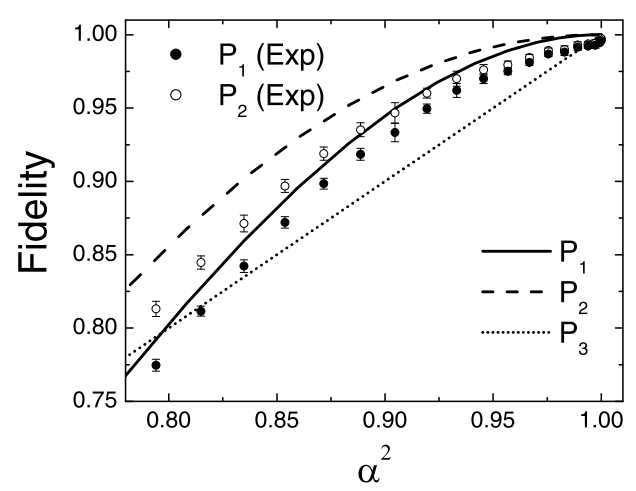

FIG. 1: Comparison of the fidelities of various protocols as a function of the parameter $\alpha^{2}$.

the inverse of $\hat{U}$; this results in the decoded state $\hat{\Phi}_{\mathbf{L}}^{(1)}=$ $\hat{U}^{\dagger}\left(|0\rangle\langle 0| \otimes \hat{\rho}_{\mathbf{L}}^{(1)}\right) \hat{U}$. The fidelity of the whole quantum coding-decoding operation for $\mathrm{P}_{1}$ is given by

$$
F_{1}=\sum_{\mathbf{L}} \frac{1}{8}\left\langle B_{\mathbf{L}}\left|\hat{\Phi}_{\mathbf{L}}^{(1)}\right| B_{\mathbf{L}}\right\rangle=\alpha^{8}\left(1+2 \beta^{2}\right)^{2} .
$$

$F_{1}$ is plotted as the solid curve in Fig. 1 and has a value of 0.9448 at $\alpha^{2}=0.9$.

The second protocol, $\mathrm{P}_{2}$, yields a higher fidelity than that of $\mathrm{P}_{1}$. In this case Alice prepares the quantum channel in the state $|00\rangle$ in the event that her projection measurement results in the state $|1\rangle$. This operation results in the average state of the quantum channel as

$$
\hat{\rho}_{\mathbf{L}}^{(2)}=\alpha^{4}\left(1+2 \beta^{2}\right)\left|\mu_{\mathbf{L}}\right\rangle\left\langle\mu_{\mathbf{L}}\left|+\beta^{4}\left(1+2 \alpha^{2}\right)\right| 00\right\rangle\langle 00| .
$$

Bob again adds an extra qubit in the state $|0\rangle$ and ap(6) plies the inverse operation $\hat{U}^{\dagger}$ to produce state $\hat{\Phi}_{\mathbf{L}}^{(2)}=$ $\hat{U}^{\dagger}\left(|0\rangle\langle 0| \otimes \hat{\rho}_{\mathbf{L}}^{(2)}\right) \hat{U}$ which has a corresponding fidelity of

$$
F_{2}=\alpha^{8}\left(1+2 \beta^{2}\right)^{2}+\alpha^{6} \beta^{4}\left(1+2 \alpha^{2}\right) .
$$

The value of $F_{2}$ is plotted as the dashed curve in Fig. 1 . $F_{2}$ has a value of 0.9652 at $\alpha^{2}=0.9$.

Finally, Jozsa and Schumacher also considered the simple protocol, $\mathrm{P}_{3}$, where Alice discards the state of every third letter and encodes the remaining letters in a block of 2 qubits, and Bob generates the state $|0\rangle$ for the missing letter state. This protocol yields an average fidelity of

$$
F_{3}=\alpha^{2},
$$

which is plotted as the dotted curve in Fig. 1.

\section{Optical scheme}

Fig. 2 shows an ideal single-photon linear optics implementation of the block coding scheme of the previous section using polarizing beam splitters, $\lambda / 2$ wave plates and 


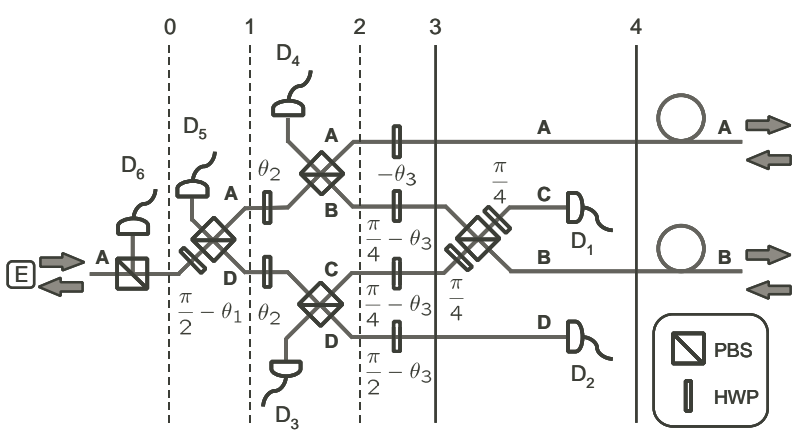

FIG. 2: Linear optics implementation of three-qubit block coding and decoding.

photodetectors. One photon is used to represent the 3 qubits in terms of two location qubits (the first 2 qubits) and a polarization qubit [4] (the third qubit). The paths labeled A, B, C and D represent the states $|00\rangle,|01\rangle$, $|10\rangle$ and $|11\rangle$ of the first two qubits, respectively, and the polarization directions in the plane and perpendicular to the plane of the optical circuit represent the states $|0\rangle$ and $|1\rangle$ of the last qubit, respectively. We parameterize the $n$th letter state by the angle $\theta_{n}=\frac{1}{2} \arcsin \left(\beta_{L_{n}}\right)$. The orientation of the fast axis of each wave plate to the vertical direction is given beside the wave plate in the figure.

First we discuss the preparation of the 3-qubit block state $\left|B_{\mathbf{L}}\right\rangle$. A horizontally-polarized photon enters the quantum circuit at position E. The first location qubit is prepared by the $\lambda / 2$ wave plate and the polarization beam splitter between vertical lines labeled 0 and 1 in terms of angle $\theta_{1}$. Similarly, the second location qubit and the polarization qubit are prepared between the vertical lines labeled 1 and 2, and 2 and 3, respectively. (Note that the photodetectors $\mathrm{D}_{3}, \mathrm{D}_{4}, \mathrm{D}_{5}$ and $\mathrm{D}_{6}$ are not used in state preparation.) The fully state-prepared block appears as a photon in a superposition of 4 path and two linear polarization modes along the vertical line labeled 3. This is the 3-qubit message Alice wants to compress and communicate to Bob.

Next we discuss the quantum coding which takes place between vertical lines 3 and 4 . The unitary transformation $\hat{U}$ is performed by the polarization beam splitter and the two $\lambda / 2$ wave plates in this section. The projection measurement of the first qubit is performed by the photodetectors $\mathrm{D}_{1}$ and $\mathrm{D}_{2}$ where the detection or non detection of a photon projects the state onto $|1\rangle$ or $|0\rangle$, respectively. The circuit in Fig. 2 implements protocol $\mathrm{P}_{1}$ explicitly: if the photodetectors $\mathrm{D}_{1}$ and $\mathrm{D}_{2}$ detect a photon no quanta will be present in the quantum channel and so the coding results in failure. The projective measurement is destructive in this case. Protocol $\mathrm{P}_{2}$ can be implemented by switching a horizontally-polarized single photon source into the optical path $\mathrm{A}$ to encode the state $|000\rangle$ each time one of the photodetectors $D_{1}$ or $\mathrm{D}_{2}$ detects a photon. The encoded (compressed) 2-qubit message appears along vertical line 4 . This is transmitted

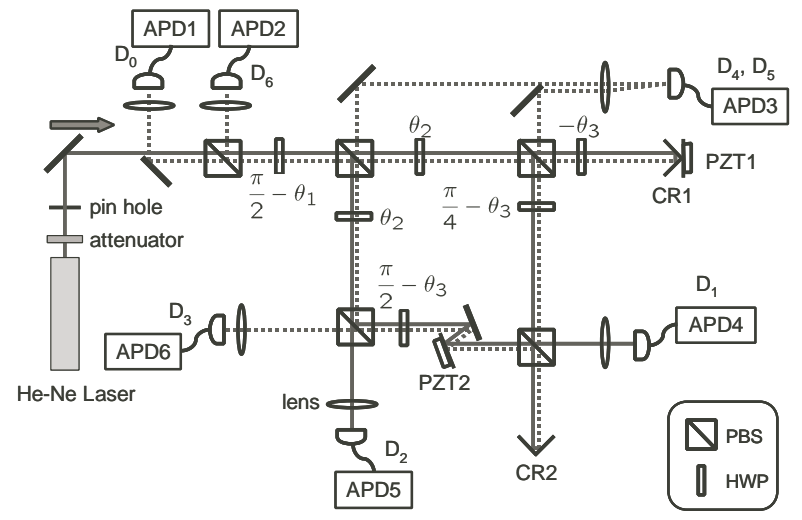

FIG. 3: Experimental setup.

to Bob.

The decoding of the quantum channel AB at Bob's site requires a mirror image of the quantum circuit in Fig. 2 between lines 3 and 4 but without the photodetectors $\mathrm{D}_{1}$ and $\mathrm{D}_{2}$. Moreover, the mirror image of the circuit to the left of the line 3 can be used to determine the fidelity of the decoded block message. The fidelity test results in a 'yes'-'no' answer for each coded-decoded block state as follows. The 'yes' answer (i.e. perfectly reconstructed letter block) is indicated by the horizontally-polarized photon emerging from the mirror image of point E. A 'no' answer is indicated by the photon being detected by one of the photodetectors $\mathrm{D}_{3}, \mathrm{D}_{4}, \mathrm{D}_{5}$ or $\mathrm{D}_{6}$ in the mirror image circuit [5].

\section{Experimental Implementation}

Our actual experimental circuit is shown in Fig. 3. Again, the orientation angle $\theta_{n}$ of each $\lambda / 2$ wave plate is given in the figure. For practical convenience, we did not construct an additional mirror-image circuit for the decoding and fidelity check. Instead we use corner reflectors (CR1 and CR2) to reflect the light in the quantum channel back through the circuit (shown as dotted lines in the figure) so that the coding and state-preparation circuits operate as decoding and state-measurement circuits for the reflected light. We use strongly attenuated light from a He-Ne laser (wavelength $632.8 \mathrm{~nm}$ ) as our single photon source. The CW laser output of $1 \mathrm{~mW}$ power is attenuated to $\approx 50 \mathrm{fW}$ which corresponds to an average photon flux of $10^{5}$ photons/sec. The average time between photons through our experiment far exceeds the time taken for light to pass through the circuit $\left(\approx 10^{-8} \mathrm{~s}\right)$.

We use multimode optical fibers with coupling efficiency of more than $80 \%$ to direct the photons exiting the circuit to silicon avalanche photodiodes (APDs). The quantum efficiency and dark count of the APDs are typically $70 \%$ and less than 100 counts/sec, respectively. The labels for each APD $\left(\mathrm{D}_{1}-\mathrm{D}_{6}\right)$ correspond to those of photodetectors in Fig. 2 and the APD labeled $\mathrm{D}_{0}$ detects the 'yes' answer of the fidelity test. Since we do not need to discriminate the photodetection between $\mathrm{D}_{4}$ and $\mathrm{D}_{5}$, we use one APD for these detectors. 


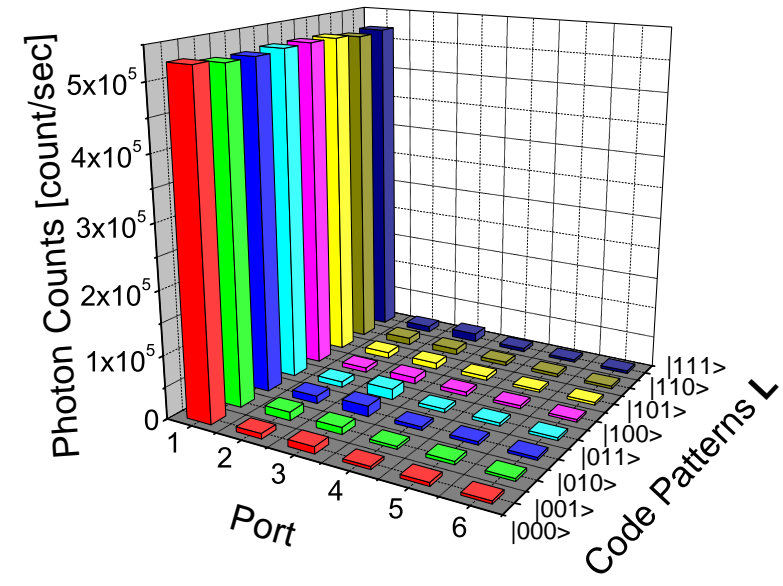

FIG. 4: Histogram of photon counts for each block state $\mathbf{L}$. The number in the vertical axis labels six APDs from APD1 to APD6. The parameter of the letter states and the fidelity are $\alpha^{2}=0.9046$ and $F=0.933 \pm 0.006$, respectively. The gating time of APDs is $5 \mathrm{sec}$.

In essence, the optical circuit consists of a Michaelson and a Mach-Zehnder interferometers controlled by Piezo transducers PZT1 and PZT2, respectively. We use a bright reference light and adjust the voltages of PZT1 and PZT2 to produce visibilities of more than $98 \%$ for these interferometers. The reference light is then switched off and the signal photons are guided into the circuit. The single photon events are counted by six APDs for each block state $\left|B_{\mathbf{L}}\right\rangle$. The gating time of the APDs is 5 sec with the combined count over $1 \mathrm{sec}$ being of the order of $10^{5}$. Since the whole apparatus is shielded by a black box, the number of background photons is much smaller than the dark count of the APDs. Also we estimate the number of events where two photons are present simultaneously in the circuit to be less than half the dark count.

Our use of a photon source with random arrival times means that the quantum coding-decoding operations occur in the context of post-selection measurements; that is, we know that a quantum coding-decoding operation has taken place after it has occurred, and, due to the limited efficiency of the photodetectors, in a subset of possible cases.

The experimental fidelity for protocol $\mathrm{P}_{1}$ is given by

$$
F_{1}^{\mathrm{ex}}=\sum_{\mathbf{L}} \frac{1}{8} \frac{N_{0}^{\mathbf{L}}}{\sum_{j=0}^{6} N_{j}^{\mathbf{L}}}
$$

where $N_{j}^{\mathbf{L}}$ is the number of photons detected by the de-

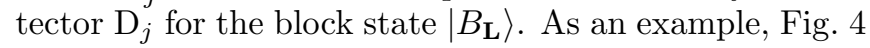
shows the photon counting data for the letter state with $\alpha^{2}=0.9046$ for which the fidelity $F=0.933 \pm 0.006$. By varying the angle $\theta$ the fidelity of our quantum codingdecoding experiment can be compared by the theoretical predictions given of the previous section over a range of $\alpha$ values. The results are shown in Fig. 1 as solid circles.

For protocol $\mathrm{P}_{2}$, rather than switching a horizontallypolarized light source into channel A each time one of the photodetectors $\mathrm{D}_{1}$ or $\mathrm{D}_{2}$ records a photon, we perform a 2 step procedure as follows. The first step is the same as for protocol $\mathrm{P}_{1}$ and, in fact, we use the same photon counting data $N_{j}^{\mathbf{L}}$ as described. The second step corresponds to the transmission of a horizontally-polarized photon in channel $\mathrm{A}$ for each of the photons detected by $\mathrm{D}_{1}$ and $\mathrm{D}_{2}$ in the first step. For this purpose, the corner reflector CR1 is removed and horizontally-polarized and attenuated light from a He-Ne laser is directed into the circuit. The number of photons used (i.e. the total number of photons detected by all APDs) in this second step is adjusted to be $N_{1}^{\mathrm{L}}+N_{2}^{\mathrm{L}}$ for each corresponding block state $\left|B_{\mathbf{L}}\right\rangle$. We can do this adjustment with an accuracy of $\pm 3 \%$ by carefully controlling the gating time of the APDs. The total fidelity for this protocol is calculated as follows:

$$
F_{2}^{\mathrm{ex}}=\sum_{\mathbf{L}} \frac{1}{8} \frac{N_{0}^{\mathbf{L}}+N_{0}^{\mathbf{L}(2)}}{\sum_{j=0}^{6} N_{j}^{\mathbf{L}}} .
$$

where $N_{0}^{\mathbf{L}(2)}$ is the total number of photons detected by $\mathrm{D}_{0}$ in the second step. We obtain the fidelities corresponding to several $\alpha$ values and plot them as open circles in Fig. 1] The experimental fidelities for both protocols exceed that of the simple protocol.

\section{Discussion}

A message of equal-likely letters is not compressible classically. In contrast, quantum source coding allows a quantum message of equally likely (but non orthogonal) letter states to be compressed [1, 2]. Our compression of 3-qubit codewords gives a clear demonstration of this fundamental principle.

The practical application of quantum source coding faces several challenges. An immediate task is to demonstrate quantum source coding using a single-photon-ondemand source. Another is to replace spatial mode qubits with frequency mode qubits as this leads to compression of bandwidth of quantum carrier. Of direct practical importance is to demonstrate the coding and decoding of a source of weak coherent states. Quantum circuits based on measurement induced non-linearities and non-classical light sources have potential in this regard. Our experiment is the first step towards realizing these practical goals.

\section{Acknowledgments}

This work was supported by the British Council, the Royal Society of Edinburgh, the Scottish Executive Education and Lifelong Learning Department and the EU 
Marie Curie Fellowship program.

[1] B. Schumacher, Phys. Rev. A51, 2738 (1995).

[2] R. Jozsa and B. Schumacher, J. Mod. Opts. 41, 2343 (1994).

[3] We perform the unitary transformation before the projection operation, otherwise our scheme is identical to that of [2].

[4] N. J. Cerf, C. Adami, and P. G. Kwiat, Phys. Rev. A 57, R1477 (1998); R. J. C. Spreeuw, Found. Phys. 28, 361

(1998); S. Takeuchi, Proc. p.299, PhysComp96 (Boston, 1996).

[5] We note that the photons arriving at $\mathrm{D}_{6}$ have only vertical polarization whereas those arriving at $D_{3}$ to $D_{5}$ can have either vertical or horizontal polarization giving, in principle, 7 distinguishable outcomes for a 'no' answer. 